\title{
On inconsistent entities. A reply to Colyvan
}

\author{
Tommaso Piazza $\cdot$ Francesco Piazza
}

Published online: 19 May 2009

(C) Springer Science+Business Media B.V. 2009

\begin{abstract}
In a recent article M. Colyvan has argued that Quinean forms of scientific realism are faced with an unexpected upshot. Realism concerning a given class of entities, along with this route to realism, can be vindicated by running an indispensability argument to the effect that the entities postulated by our best scientific theories exist. Colyvan observes that among our best scientific theories some are inconsistent, and so concludes that, by resorting to the very same argument, we may incur a commitment to inconsistent entities. Colyvan's argument could be interpreted, and in part is presented, as a reductio of Quinean scientific realism; yet, Colyvan in the end manifests some willingness to bite the bullet, and provides some reasons why we shouldn't feel too uncomfortable with those entities. In this paper we wish to indicate a way out to the scientific realist, by arguing that no indispensability argument of the kind suggested by Colyvan is actually available. To begin with, in order to run such an indispensability argument we should be justified in believing that an inconsistent theory is true; yet, in so far as the logic we accept is a consistent one it is arguable that our epistemic predicament could not be possibly one in which we are justified in so believing. Moreover, also if our logic admitted true contradictions, as Dialetheism does, it is arguable that Colyvan's indispensability argument could not rest on a true premise. As we will try to show, dialetheists do not admit true contradictions for cheap: they do so just as a way out of paradox, namely whenever we are second-level ignorant as to the metaphysical possibility of evidence breaking the parity among two or more inconsistent claims;
\end{abstract}

This paper is an outcome of the S\&MIE project.

T. Piazza $(\bowtie)$

Faculdade de Letras, University of Porto, Rua Panoramica s/n, 4150-564 Porto, Portugal e-mail: tpiazza@letras.up.pt

\section{F. Piazza}

Ecole Polytechnique Fédérale de Lausanne (EPFL), Lausanne 1015, Switzerland e-mail: francesco.piazza@epfl.ch 
Colyvan's examples, however, are not of this nature. So, even under the generous assumption that Dialetheism is true, we will conclude that Colyvan's argument doesn't achieve its surprising conclusion.

Keywords Ontological commitment · Indispensability argument ·

Realism · Inconsistent entities · Dialetheism

Scientific realists, as contrasted with scientific anti-realists, believe that our best scientific theories, at least in the most favourable cases, tell us something true about a mind-independent and unobservable reality. Many arguments have been advanced to vindicate the latter claim; yet one, originally formulated by $\mathrm{H}$. Putnam as the nomiracles argument, occupies a prominent position in the literature: according to this argument, the empirical and predictive success of our best scientific theories would be utterly inexplicable if we did not assume that these theories, above and beyond being empirically adequate, indeed truly describe the functioning of nature. Since most of our theories indeed enjoy a big deal of predictive success, therefore, we are forced to assume that they are true.

A step further, which the scientific realist is normally willing to take, involves the transition from the belief in the truth of our best and more successful theories to the belief in the existence of the entities to which these theories makes reference. This path from truth to existence, marking what has come to be known as the Quinean way to metaphysics, give raise to many indispensability arguments. Indeed, the general suggestion is that whenever we have reasons to believe in a theory we should not stop short of acknowledging the existence of the entities over which the theory quantifies (in a non-eliminable way). Usual first-order semantic machinery sustains this move: a theory involving singular reference to a given entity cannot be true unless the entity exists in the very first place; so, if no suitable reformulation of a theory is available which is at least as attractive as the first theory (Colyvan 2008 b), yet avoid making reference to the entities in question, every reason we have for accepting the theory is a reason for accepting the entity. This has been argued not only in the case of the unobservable entities postulated by our best theoretical science; some authors have also contended that the very existence of numbers could be inferred by the observation that mathematics is indispensable for our best scientific practice, and the apparent datum that mathematical claims indeed involve reference to numbers. Most recently, however, M. Colyvan has argued that an indispensability argument could be mounted also to vindicate the existence of entities whose metaphysical extravagance far exceeds that of abstract entities, namely that of inconsistent objects. In this paper we wish to examine Colyvan's surprising argument, and to assess whether the reasons it gives for accepting its prima facie bewildering conclusion survive philosophical scrutiny. ${ }^{1}$

\footnotetext{
${ }^{1}$ It is fair to admit that Colyvan pursues a more nuanced strategy. Colyvan's first aim is to establish a conditional claim: if we allow an indispensability argument to tell us what there is, then we are committed
} 
Colyvan begins by offering empirical evidence for the claim that our best science contains inconsistent theories. Notoriously, observes Colyvan, contemporary science accepts two theories which contradict each other, namely the Theory of Relativity and Quantum Mechanics. However, he goes on to argue, examples of inconsistent theories are also of more mundane nature. In particular he gives the following examples: (a) the Newtonian Gravitational theory, which gives a certain prediction for the rotation of spiral galaxies, is inconsistent with the "theory involved in determining the radial speed of galaxies (Doppler shifts)" (2008a, p. 117), as the rotation thereby observed is different than the theoretically predicted one; (b) the theory of waves, which assumes infinitely deep oceans, is contradicted by "relevant pieces of theory that give finite depths to oceans" (ibidem); (c) the theory of infinitesimals, as developed between the seventeenth and the nineteenth century, which over a century has both held infinitesimals to be quantities different-than and equal-to zero; (d) naïve set-theory, notoriously shown by Russell to entail a contradiction.

Since Colyvan's aim is to show that an indispensability argument having as its conclusion the existence of inconsistent entities could be mounted which resembles in all relevant respects more uncontroversial indispensability arguments, it seems reasonable to expect that the examples he puts forward be based on inconsistent theories which our current scientific community regards as true. However, Colyvan never says in explicit terms that, besides being among our best scientific theories, we have reasons to believe that the theories he proposes as examples are true; moreover, no example seems to be possibly of this nature. ${ }^{2}$

Example (d), most prominently, is an example of an inconsistent theory which has been regarded as true until its inconsistency has been revealed by Russell: as a consequence, it is clearly an unserviceable premise for an indispensability argument having as its conclusion the existence of an inconsistent object, namely the set of all sets which do not belong to their-selves. Much to the contrary, the theory's being

Footnote 1 continued

to swallowing the conclusion that there are inconsistent entities. Secondly, Colyvan addresses the question about how the scientific realist should react to the latter claim. It is only at this point that Colyvan advances the suggestion that the realist should bite the bullet, and let the Quinean strategy deposit within our ontology, among other things, also inconsistent entities. In this paper we expressly address Colyvan's second contention. However, what we say is by the same token meant to oppose his first conditional claim.

${ }^{2}$ It might be observed, right from the start, that an indispensability argument to the existence of a given class of entities does not need as a premise the claim that the theory which postulates these entities is true. All that is needed is the weaker claim that we have reasons to believe in an inconsistent theory. Once this premise is in place, the indispensability argument just tells us that, by the same token, we also have reasons to believe in the entities postulated by the theory. We agree with the latter contention; however, we also doubt that in the present context the premise under consideration is any weaker than the first one. In order to be entitled to run an argument having as a premise the claim that $p$ is true, in fact, all that is needed is to have reasons to believe that $p$. Unless the notion of a belief is not used idiosyncratically, to believe that $p$ is to believe that $p$ is true, and so any reason for the belief that $p$ is by the same token a reason for the belief that $p$ is true. Moreover, it should be stressed that the notion of a belief could not, in the present context, be used idiosyncratically: did we "believe" in a theory, but endorse no commitment concerning its truth, we would be entitled to draw no inference concerning the existence of the entities it postulates. 
inconsistent has been regarded as a sufficient motivation for not regarding the theory as true in the very first place. ${ }^{3}$ The same considerations militate against example (c): the calculus of infinitesimals is no longer accepted in its original inconsistent formulation; quite to the contrary, it is owing to the work of Bolzano and others, which removed the contradiction, that the calculus is nowadays regarded as a serviceable mathematical tool indispensable to scientific practice.

Example (b), in turn, also seems of doubtful compatibility with Colyvan's purposes. On the one hand, it might be argued, the assumption that oceans have infinite depths is a theoretical idealization and so, as such, it is without any real empirical content; moreover, also if it were correct to construe the idealization concerning the infinite depth of the oceans as an empirical claim, and so to construe its logical relationship with the empirical observation that oceans are finite in depth as a contradiction, it is doubtful that the upshot should be the one suggested by Colyvan: if our best theory really implied that the oceans have infinite depths, well, the worse for this theory; standard Sonar techniques reveal that the oceans do not. Also in this case we would not have any contradiction: much in the spirit of N. Cartwright's wellknown criticism of contemporary science (1983), we would just be revealed that the idealisation concerning the oceans' depth, much like every other idealisation interpreted as an empirical claim, just fails to convey something true of reality. ${ }^{4}$

\footnotetext{
${ }^{3}$ At a certain moment Colyvan summarizes the significance of his argument by recapitulating its conclusion as follows: "there were times when we had warrant to believe in [...] inconsistent objects" (p. 119). As it is clear, this claim is weaker than the claim that we (now) have reasons to believe that there are inconsistent entities; however, example (d) is arguably also not sufficient to establish it. Let us grant the point, which is not necessarily uncontroversial, that there have been times in which we were justified in believing in Frege's naïve set-theory (every theorist uncomfortable with the claim that a priori justification is defeasible would deny this). By the fact that naïve set theory is inconsistent, and so, if true, committal to inconsistent entities, it by no means follows that there have been times in which we were justified in believing that there are inconsistent entities. The reason is that justification is in general not closed under (unknown) logical implication: until the contradiction has not been revealed by Russell, no one knew of the inconsistent consequences of naïve set theory, and so the evidence then available did not transfer to the belief in those consequences. Moreover, and more in particular, it is also arguable that justification is not closed when the deliverance of logical implication, as in the case at issue, is defeating evidence. As a matter of fact, the discovery of the inconsistent consequences of the theory has been taken to show that when accepting the theory we were wrong, and that the evidence we had used to rely upon was misleading.

${ }^{4}$ It is surprising that Colyvan doesn't mention this possible interpretation of the assumption that oceans are infinitely deep. As a matter of fact he explicitly derives the example from Maddy (1992), where it is expressly recognized that mathematical idealizations of this sort, although they play a fundamental theoretical role, say of reality something which is literarily false: "[The] pages [of a freshmen physics text] are littered with applications of mathematics that are expressly understood not to be literally true: e.g., the analysis of water waves by assuming the water to be infinitely deep or the treatment of matter as continuous in fluid dynamics or the representation of energy as a continuously varying quantity. Notice that this merely useful mathematics is still indispensable; without these (false) assumptions, the theory becomes unworkable" (Maddy 1992, p. 281). Maybe Colyvan, by suggesting that our best science characterizes oceans both as infinitely and finitely deep, is driven by the thought that a Quinean realist has not the resources to tell fundamental, but literally untrue parts of science apart from fundamental and literally true parts of science. By so doing, however, he would be advancing the contention that the Quinean realist could not use the no-miracle argument to ground the contention that a given theory is true, and at the same time avoid the commitment to inconsistent entities mediated by the corresponding indispensability argument. However, it is clear that the Quinean realist could avoid this upshot by simply dropping the no-miracle argument, and by grounding her belief in the truth of a given theory in a different way.
} 
As far as example (a) is concerned, there also seems to be grounds for rejecting Colyvan's allegation that the conflict it exposes "can be seen for what it is: a contradiction" (2008a, p. 116). As a matter of fact, Colyvan's presentation of the debate is a gross simplification. Colyvan is right in reporting that in many cases the rotation curves of spiral galaxies reconstructed by spectroscopic methods reveal much greater velocities in the disk regions than those predicted by Newton's law of gravitation, and that, in particular, the measured rotation curves are not in agreement with those implied by luminosity. However, these results have not led astronomers to believe that spiral galaxies have inconsistent masses; quite to the contrary, they have led them to hypothesize that spiral galaxies are embedded in extended halos of dark matter, which surrounds galaxies but is transparent to all forms of electromagnetic radiation. By adverting to dark matter contemporary physicists restore consistency among the data: if spiral galaxies are embedded in dark matter, the mass measures implied by luminosity will lead to the prediction of velocities which are inconsistent with those revealed by measured rotation curves. The majority of them, ${ }^{5}$ then, believe that Newtonian laws hold true, and just believe luminosity to be an imprecise indication of mass. Accordingly, we seem allowed to conclude that the presence of a( $\mathrm{n}$ apparent) conflict among Newtonian gravitational theory and the observed rotation of spiral galaxies is not taken to deliver some warrant for accepting, as true, the (inconsistent) conjunction of both the theory and the observation. Therefore, also in this case no indispensability argument of the sort envisaged by Colyvan seems to be available.

Moreover, much independently of what contemporary scientists actually do, there seem to be general reasons for supposing that they should, from an epistemic point of view, act in this way (and therefore, for supposing that no indispensability argument should be available in this case). In fact, it seems sensible to suppose that contemporary scientists could not have grounds to accept as true a theory or a conjunction of theories if, as in the present case, this theory or conjunction of theories is inconsistent: its being inconsistent, in fact, prevents it from being true as a matter of logic.

This observation, it could be argued, makes available a common recipe by using which every possible indispensability argument having as its conclusion the claim that some inconsistent entities exist could be rejected, as it were, by definition. The very simple idea is that any sound indispensability argument needs to feature as a premise a claim to the effect that (we are justified in believing that) a given theory, involving reference to some entities, is true, if this argument is to ground the conclusion that those entities exist; however, if the claim to be established is that some inconsistent entities exist, then no such premise can ever be available, because

\footnotetext{
5 There are also different schools of thought that challenge Newton laws as they are derived in the weak gravitational limit from Einstein's general relativity (GR). Hartnett (2005), for example, has shown that Carmeli's five-dimensional cosmology (generalizing GR by accounting for Hubble flow of matter) seems to explain the actual rotational curves of galaxies without the need of bringing dark matter into the picture. It is fair to admit that the latter solution is considered somewhat heretical by the community of physicists, generally preferring admitting dark matter over departing from well-established scientific paradigms as Newtonian Gravitational Theory. However, it also testifies to the unwillingness of the current scientific community to countenance, as admissible, inconsistent solutions to the problem at issue.
} 
that premise should be to the effect that (we are justified in believing that) an inconsistent theory is true, whereas no inconsistent theory, as a matter of logic, can be. This, at bottom, is the reason why all the examples put forward by Colyvan seem to fail: examples (c) and (d), more straightforwardly, because they involve reference to theories which are currently regarded as false because of their inconsistency; examples (a) and (b) because they involve reference to theories, or conjunctions of theories, which no one ought to regard as true because of their inconsistencies.

To be sure, this reply substantially rests on an epistemic principle to the effect that a person who is aware that a given proposition (or set of propositions) is inconsistent is, by the same token, indefeasibly justified in believing that this proposition (or set of propositions) is false. Now, we seriously doubt that there is an epistemologist or a philosopher of science which would deny this. However, we are not advocating an argument ex autoritate. Moreover, it is fair to admit that there are some logicians which would deny it. Accordingly, we shall devote the reminder of this reply to taking in consideration a line of resistance, emerging from some sparse remarks in Colyvan's paper, which draws on logic revision. As we will try to show, by advocating logical revision Colyvan could consistently deny the abovementioned principle, and so avail himself of the claim that some contradictions may be rationally believed to be true. However, as we shall try to argue, the motivations available for the needed logical revision hardly recommend the conclusion that we might be justified, in particular, in accepting the contradictions allegedly surfacing in Colyvan's examples.

In addressing some possible objections Colyvan concentrates, among the others, to the following two possible retorts: (i) trivialisation: once you accept a contradiction, then the logical principle ex contraductione quodlibet commits you to accepting whichever proposition; therefore, on pains of trivialising the notion of justification, a subject cannot be justified in accepting a contradiction (2008a, p. 120); (ii) theoretical virtuousness: being justified is a matter of deploying theoretical virtues; consistency is the highest theoretical virtue; therefore, a subject cannot be justified in entertaining an inconsistent proposition (2008a, p. 120). Both retorts seem to ground something in the vicinity of the principle that a person is always justified in disbelieving an inconsistent proposition; therefore, in the present context, it seems recommendable to concentrate on Colyvan's answer to both.

As against (i) Colyvan correctly observes that trivialisation ensues just if one accepts an explosive logic, namely a logic which incorporates the principle in question. However, if one accepts a (weak or strong) paraconsistent logic, which denies it, no problem of trivialisation is anymore forthcoming. As against (ii), much in the same spirit, Colyvan denies that consistency should be credited a privileged place among our theoretical virtues; for the simple reason that how consistency is to be ranked seems to depend on whether one's underlying logic allows or not some contradictions to be true, and some logics, notably Dialetheism, do. Accordingly, Colyvan's answer to the general reply we have been arguing in the preceding 
section might simply be that, provided one's logic is of the appropriate sort, it is simply false that a person can never be justified in accepting a proposition (or set of propositions) which she knows to be inconsistent. Since the difference between a strongly paraconsistent logic, as Dialetheism, and a weakly paraconsistent logic is that just the former both allows some contradictions to be true and denies the principle ex contraductione quodlibet (whereas a weakly paraconsistent logic may just deny the latter principle) in what follows we shall concentrate on Dialetheism. As the issue whether Dialetheism is correct far exceeds the limited scope of this paper, we shall not directly address it, though. Rather, we shall concentrate on the conditional claim, which seems to underlie Colyvan's possible reply, that if Dialetheism is correct, then (at least some of) his examples could be used to mount an indispensability argument to the existence of inconsistent entities. For the purposes at hands, this will suffice; for we also believe, and shall argue, that even granting to Dialetheism no less than the strongest possible case in its favour we achieve no perspective from which the contradictions exposed by Colyvan could be shown to be actual dialetheias which we have reasons to accept.

To begin with, as the following quote shows, it should be recalled that Dialetheism is the view that some contradictions are true, and, in addition, that the evidence in our possession may sometimes justify us in accepting some contradiction:

In this chapter I will address the title question [What's so bad about contradictions?]; and the answer I shall give is 'maybe nothing much'. Let me first explain how, exactly, the question is to be understood. I shall interpret it to mean 'what is wrong with believing some contradictions?' I emphasize the 'some'; the question 'what is wrong with believing all contradictions' is quite different, and, I am sure, has a quite different answer. It would be irrational to believe that I am a fried egg [...]. A fortiori, it is irrational to believe that I am both a fried egg and not a fried egg. It is important to emphasize this distinction right at the start, since the illicit slide between 'some' and 'all' is endemic in discussion of the question [...] (Priest et al. 2004, p. 23)

Dialetheism, as R. Sainsbury has nicely put the point, is then the view that "some contradictions are true" and that "for some contradictions, it is rational to believe that they are true" (1995, p. 135). Given its dual nature, Sainsbury has coined the label "rational Dialetheism" for the conjunction of both views, and reserved "dialetheism" just to the position that there are-no matter whether they are knowable-true contradictions. Put in this way, Colyvan's conditional claim may be paraphrased as follows: if rational dialetheism is true, we have reason to suppose that some inconsistent scientific theory-for example, the conjunction of Newtonian gravitational theory, and the theory involved in determining the mass-density of a galaxy-is true. In what follows we shall try and assess this conditional claim: firstly, we shall analyse the strongest possible case in favour of rational dialetheism; secondly, we shall diagnose the nature of this case, and so state in general terms the conditions under which, according to the rational dialetheist, it is rational to accept a contradiction; thirdly, we shall argue that those conditions are notably not satisfied in the case of Colyvan's inconsistent theories; having done so, we shall conclude 
that the strongest possible evidence in favour of rational dialetheism doesn't sustain the conjecture that Colyvan's inconsistent theories are true, and so on this basis, we shall reject the conditional claim.

Commenting on the twentieth century renaissance of dialetheism Priest and Berto (2008) have recently maintained:

Turning now to contemporary philosophy, the second half of the twentieth century saw a resurgence of dialetheism, driven by largely new considerations. Probably the major argument used by modern dialetheists invokes the logical paradoxes of self-reference.

The rough idea is that, if at all plausible, dialetheism can be vindicated in light of its superior capability with respect to rival theories to cope with the semantic and set-theoretic paradoxes (see also Sainsbury 1995, p. 144). Take the Liar paradox, in the following form:

\section{(1) What (1) says is false.}

The paradox derives from the fact that if we take (1) to be saying something true, then we are forced by an apparently sound argument to take (1) to be saying something false; and if we take (1) to be saying something false, then we are forced by another apparently sound argument to take it to be saying something true. Unlike many rival accounts, dialethism takes this evidence at face-value, and accepts on this basis that (1) is both true and false. In this case, as Priest explicitly recognizes, the inconsistent conclusion is arrived at by weighting several epistemic considerations, and by sacrificing consistency to other theoretical virtues such as simplicity, non-adhocness, and problem-solving ability. The idea, then, is that believing in a contradiction may be rational only provided that "the combined force of other criteria" is enough to trump consistency (2004, p. 32), a situation which is characteristically exemplified by the paradox at issue: the alternative solutions, so contends the dialetheist, "all have the virtue of consistency" but "often have strong ad hoc elements; they are complex, often involving transfinite hierarchies; they have a tendency to pose just as many problems as they solve; and it is not clear that, in the last instance, they really solve the problem they are supposed to" (p. 33).

The rational dialetheist, to be sure, does not stay content with the suggestion that true contradictions just "arise in the relatively abstract realm of logic" (Priest 1987, p. 200). Much to the contrary, contemporary dialetheists also contend that true contradictions reach well out in the empirical domain within which Colyvan's examples find their proper location. In other words, they believe that rational dialetheism can be established also in light of cases arising from the empirical world. However, also in this case the rational dialetheist ultimately contends that our justification in accepting a contradiction derives from its providing the best way out to paradox. For instance, it is suggested that rational dialetheism could be established in light of the presence, within ordinary language, of vague predicates: only by admitting that a thing may at the same time satisfy and fail to satisfy any such predicate, contends the rational dialetheist, we may end up with a satisfactory way out to the paradox of the Sorites; therefore, we have to admit it (for details on the so called sub-valuationist approach to vagueness, see Hyde 1997). 
These examples should suffice for a principled statement of the conditions under which, according to the rational dialetheist, we should regard a contradiction as one which we are justified in accepting. What all the cases just envisaged have in common, it could be suggested, is that true contradictions are (correctly or incorrectly) posited whenever (correctly or incorrectly) it is judged that no other equally serviceable solution is available. To wit: we might be wrong in rejecting available alternatives, or in believing that no presently ignored account might provide a consistent way out to paradox; however, it is not a priori certain that we are wrong, nor that there must be a consistent alternative: this is guaranteed by the "dialetheism" in rational dialetheism, namely by the contention that there are true contradictions; accordingly, as far as our present state of information warrants the belief that no consistent solution will do as good as the consistent one, our state of information warrants the belief in a contradiction. To emphasize the point, just consider how Armour-Garb and Woodbridge (2006, p. 401) describe the rational dialetheist's methodological predicament with respect to the Liar:

The dialetheist recognizes that if a consistent approach could resolve the semantic paradoxes without falling victim of the aforementioned pitfalls, then, on methodological grounds, he would have reasons to opt for that approach. Of course the dialetheist predicts that no such resolution will be forthcoming, and he therefore does not pursue such an approach. Instead he [...] defends the necessary logical revision [...]

Now, the standing reference to paradox, surfacing in the account just presented, should not be underestimated. One might be tempted to read the tentative view outlined above concerning the conditions under which it is rational to accept a contradiction as the view that it is rational to do so whenever we are faced with one or more inconsistent statements, and the evidence at our disposal equally well supports each of them. Actually, according to the dialetheist the paradoxes reviewed so far have this feature, as she alleges that the justification we have for accepting each premise responsible for the contradiction is not defeated by any motivation underlying alternative, and consistent approaches. However, it must be stressed that the dialetheist's predicament, as Armour-Garb and Woodbridge nicely suggest, is also characterized by an additional crucial feature: the (allegedly) justified belief that no evidence defeating the justification we presently have for one or more of the premises is actually forthcoming. This feature, it might be suggested, is actually constitutive of a paradox, and it is for this reason that no less than paradox, according to the rational dialetheist, could rationally impose the acceptation of a contradiction. ${ }^{6}$ Whenever we face an inconsistent pair, and our meta-epistemic

\footnotetext{
${ }^{6}$ On its most common usage the label "paradox" is used to indicate a claim, or a set of claims, which leads to a contradiction. So understood, every situation in which we have a pair of inconsistent statements $S_{1}$ and $S_{2}$ such that the evidence in our possession favours both statements should be regarded as one in which we are faced with a paradox. Under this reading, needless to say, also Colyvan's examples could be read as paradoxes. However, it is fair to admit that we often find ourselves in situations of the envisaged sort without feeling the slightest inclination to regard ourselves as facing a paradox. Mr Smith told you that he's going to join the afternoon meeting, while two colleagues of yours maintain that Mr Smith told them he is not. Assuming you're knowledgeable that both Mr Smith and the two colleagues are reliable, and that no other available consideration allows you to attribute to either testimony more credibility,
} 
predicament is not of the kind just envisaged-either because we have reasons for expecting new evidence breaking the parity, or we simply do not have reason either way, neither for expecting new evidence nor for believing that it is not forthcoming-we should not regard ourselves as being faced with a true contradiction.

Now it is doubtful that the cases envisaged by Colyvan exhibit the features which the rational dialetheist regards as necessary for their true contradictoriness to prove an acceptable alternative. This is quite straightforward the case for examples (b), (c) and (d). As already seen, example (d) is about a theory which has actually been rejected owing to its contradictoriness, a serviceable alternative having proved the one of developing less-than-naïve and consistent set theory; example (c), in the same fashion, is about a theory which turned out to admit of a consistent foundation; example (b), in turn, can actually be treated by way of conceptually clarifying the actual contribution which literally false mathematical idealizations play in fundamental science: in order to dispel the appearance of a true contradiction it is actually sufficient to bear in mind N. Cartwright's teachings. The situation doesn't seem to be different, even if for different reasons, in the case of the first example, and, finally, in the case of the less mundane conflict among our current major theories, the Theory of Relativity and Quantum Mechanics.

Arguably, the conflicts which pervade those (conjunctions of) theories have no more significance, for contemporary scientists, than simply an indication of the fact that further investigation, new experiments and better theorizing are needed to overcome present difficulties. This is how science has proceeded so far: the history of science is littered with examples of clashes among theories and different parts of a single theory. However, progress has always been made by addressing those conflicts and by way of finding consistent theoretical accommodations to them. So, even on an inductive ground, it is not clear why contemporary scientists should regard themselves as having no other alternative but accepting that reality behaves contradictorily.

\footnotetext{
Footnote 6 continued

you're faced with two equally justified and inconsistent claims, that Mr Smith is coming, and that he isn't. However, you don't believe you've found a novel paradox, the paradox of Mr Smith. Rather you just wait for novel evidence to break the parity, one way or another. What is distinctive of a paradox, then, seems to be that this third way-waiting for novel evidence-is (or can be shown to be) on a principled basis unavailable, just because the situation seems to be one in which you're justified in believing that no novel evidence is forthcoming. What is needed, then, is that your predicament be characterised by second-level ignorance, namely ignorance as to the metaphysical possibility of there being possible evidence of the required sort in the very first place. Borderline cases of vague predicates, for instance, seem to fit this characterization. As C. Wright has written, dwelling on the epistemic predicament of a subject faced with a borderline case of red, in this situation "it is also not known whether knowledge, one way or another, about the redness of a particular case is even metaphysically possible-whether there is metaphysical space, so to speak, for such an opinion to constitute knowledge (Wright 2001, p. 75). Arguably, this somewhat regimented use of the label "paradox" is not one which R. M. Sainsbury would accept. In Sainsbury (1995, p. 1) a paradox is defined as "an apparently unacceptable conclusion deriving by apparently acceptable reasoning from apparently acceptable premises". However, Sainsbury adds that "paradoxes come in degree, depending on how well appearance camouflages reality": accordingly, our use of "paradox" could then be taken to pick out only those paradoxes about which available evidence warrants the belief that the appearances should be taken at face value, as revealing, rather than camouflaging, reality.
} 
To conclude: so far we have defended the claim that no sound indispensability argument can be mounted to the effect that our current best science commits us to acknowledging the existence of inconsistent entities. If we accept a consistent logic, this is true almost by definition, as an indispensability argument needs as a major premise a claim to the effect that (we are justified in believing that) a given theory is true, and under that assumption no belief in an inconsistent scientific theory is possibly justified, as a matter of logic. Even if we accept an inconsistent logic, and so keep open-minded about true contradictions, however, we have arguably shown that we have in particular no reasons to accept Colyvan's theories as examples of true scientific contradictions.

As it is clear, if Rational Dialetheism is correct, then there are true contradictions we are justified in accepting, and so, after all, some indispensability argument having the conclusion that some inconsistent entities exist can be mounted. Whether it is so, however, actually depends on whether Dialetheism, or something like it under the relevant respects, could be vindicated. Only at that condition we could accept an indispensability argument to inconsistent entities. Unfortunately, we have not the space to address this question here. By now, then, it seems advisable to stay content just with the more modest conclusion that for all that Colyvan has shown to us, we are actually justified in accepting none.

\section{References}

Armour-Garb, B., \& Woodbridge, J. A. (2006). Dialetheism, semantic pathology, and the open pair. Australasian Journal of Philosophy, 84(3), 395-416. doi:10.1080/00048400600895912.

Cartwright, N. (1983). How the laws of physics lie. Oxford: Clarendon Press.

Colyvan, M. (2008a). The ontological commitment of inconsistent theories. Philosophical Studies, 141, 115-123. doi:10.1007/s11098-008-9266-5.

Colyvan, M. (2008b) Indispensability arguments in the philosophy of mathematics. Stanford Encyclopaedia of Philosophy, http://plato.stanford.edu/entries/mathphil-indis/\#1

Hartnett, J. G. (2005). Spiral galaxy rotation curves determined from Carmelian general relativity. International Journal of Theoretical Physics, 44(3), 349-362. doi:10.1007/s10773-005-3366-1.

Hyde, D. (1997). From heaps and gaps to heaps of gluts. Mind, 106(424), 641-660. doi: 10.1093/mind/106.424.641.

Maddy, P. (1992). Indispensability and practice. The Journal of Philosophy, 89(6), 275-289. doi: $10.2307 / 2026712$.

Priest, G. (1987). In contradiction. A study in the transconsistent. Dordrecht: Martinus Nijhoff.

Priest, G., Beall, J. C., \& Armour-Garb, B. (2004). The law of non contradiction. New philosophical essays. Oxford: Oxford University Press.

Priest, G., \& Berto, F. (2008). Dialetheism. Stanford Encyclopaedia of Philosophy, http://plato.stanford. edu/entries/dialetheism/\#3.3

Sainsbury, R. M. (1995). Paradoxes. Cambridge: Cambridge University Press.

Wright, C. (2001). On being in a quandary. Relativism vagueness logical revision. Mind, 110(437), 45-98. 